\title{
The influence of bright and dim light on substrate metabolism, energy expenditure and thermoregulation in insulin-resistant individuals depends on time of day
}

\author{
Jan-Frieder Harmsen ${ }^{1}$ (D) Jakob Wefers ${ }^{1} \cdot$ Daniel Doligkeit $^{1}$ (D) Luc Schlangen $^{2}$ (D) Bas Dautzenberg $^{1}$. \\ Pascal Rense ${ }^{1}$ (D) - Dirk van Moorsel ${ }^{3}$ - Joris Hoeks ${ }^{1}$ (D) - Esther Moonen-Kornips ${ }^{1}$ - Marijke C. M. Gordijn ${ }^{4,5}$. \\ Wouter D. van Marken Lichtenbelt ${ }^{1}$ • Patrick Schrauwen ${ }^{1}$ (B)
}

Received: 2 July 2021 / Accepted: 22 October 2021 / Published online: 2 February 2022

(C) The Author(s) 2022

\begin{abstract}
Aims/hypothesis In our modern society, artificial light is available around the clock and most people expose themselves to electrical light and light-emissive screens during the dark period of the natural light/dark cycle. Such suboptimal lighting conditions have been associated with adverse metabolic effects, and redesigning indoor lighting conditions to mimic the natural light/dark cycle more closely holds promise to improve metabolic health. Our objective was to compare metabolic responses to lighting conditions that resemble the natural light/dark cycle in contrast to suboptimal lighting in individuals at risk of developing metabolic diseases.

Methods Therefore, we here performed a non-blinded, randomised, controlled, crossover trial in which overweight insulin-resistant volunteers $(n=14)$ were exposed to two $40 \mathrm{~h}$ laboratory sessions with different $24 \mathrm{~h}$ lighting protocols while staying in a metabolic chamber under real-life conditions. In the Bright day-Dim evening condition, volunteers were exposed to electric bright light $(\sim 1250 \mathrm{~lx})$ during the daytime $(08: 00-18: 00 \mathrm{~h})$ and to dim light $(\sim 5 \mathrm{~lx})$ during the evening $(18: 00-23: 00 \mathrm{~h})$. Vice versa, in the Dim day-Bright evening condition, volunteers were exposed to dim light during the daytime and bright light during the evening. Randomisation and allocation to light conditions were carried out by sequential numbering. During both lighting protocols, we performed $24 \mathrm{~h}$ indirect calorimetry, and continuous core body and skin temperature measurements, and took frequent blood samples. The primary outcome was plasma glucose focusing on the pre- and postprandial periods of the intervention.

Results Spending the day in bright light resulted in a greater increase in postprandial triacylglycerol levels following breakfast, but lower glucose levels preceding the dinner meal at 18:00 h, compared with dim light $(5.0 \pm 0.2 \mathrm{vs} 5.2 \pm 0.2 \mathrm{mmol} / \mathrm{l}, n=13, p=0.02)$. Dim day-Bright evening reduced the increase in postprandial glucose after dinner compared with Bright day-Dim evening (incremental AUC: $307 \pm 55$ vs $394 \pm 66 \mathrm{mmol} / 1 \times \min , n=13, p=0.009)$. After the Bright day-Dim evening condition the sleeping metabolic rate was identical compared with the baseline night, whereas it dropped after Dim day-Bright evening. Melatonin secretion in the evening was strongly suppressed for Dim day-Bright evening but not for Bright day-Dim evening. Distal skin temperature for Bright day-Dim evening was lower at $18: 00 \mathrm{~h}\left(28.8 \pm 0.3^{\circ} \mathrm{C}\right.$ vs $\left.29.9 \pm 0.4^{\circ} \mathrm{C}, n=13, p=0.039\right)$ and higher at 23:00 $\mathrm{h}$ compared with Dim day-Bright evening $\left(30.1 \pm 0.3^{\circ} \mathrm{C}\right.$ vs $\left.28.8 \pm 0.3^{\circ} \mathrm{C}, n=13, p=0.006\right)$. Fasting and postprandial plasma insulin levels and the respiratory exchange ratio were not different between the two lighting protocols at any time.
\end{abstract}

Jan-Frieder Harmsen and Jakob Wefers contributed equally to this study. Wouter van Marken Lichtenbelt and Patrick Schrauwen share last authorship.

Patrick Schrauwen

p.schrauwen@maastrichtuniversity.nl

1 Department of Nutrition and Movement Sciences, NUTRIM School for Nutrition and Translational Research in Metabolism, Maastricht University Medical Center, Maastricht, the Netherlands

2 Human-Technology Interaction Group and Intelligent Lighting Institute, Department of Industrial Engineering and Innovation Sciences, Eindhoven University of Technology, Eindhoven, the Netherlands
3 Division of Endocrinology, Department of Internal Medicine, Maastricht University Medical Center, Maastricht, the Netherlands

4 Chronobiology Unit, Groningen Institute for Evolutionary Life Sciences, University of Groningen, Groningen, the Netherlands

5 Chrono@Work, Groningen, the Netherlands 


\section{Research in context}

\section{What is already known about this subject?}

- Light is the most important time cue to synchronise the circadian clock to the environmental $24 \mathrm{~h}$ light/dark cycle

- Light at night affects glucose metabolism and is associated with the development of overweight, obesity and cardiovascular disease in humans

- Bright light in the morning influences postprandial metabolism in healthy volunteers but also in type 2 diabetes patients

\section{What is the key question?}

- Does optimised artificial indoor lighting that mimics the natural light/dark cycle more closely—characterised by bright light during the daytime and dim light during the evening-elicit more favourable metabolic effects in insulin-resistant volunteers in contrast to the opposite lighting pattern?

\section{What are the new findings?}

- Bright day-Dim evening mostly elicited favourable outcomes: lower plasma glucose levels preceding the dinner meal, greater energy expenditure in response to the dinner meal and facilitation of a diurnal pattern in distal skin temperature

- The sleeping metabolic rate dropped within the Dim day-Bright evening condition, whereas it was conserved upon Bright day-Dim evening

- Dim day-Bright evening reduced postprandial triacylglycerol levels after breakfast and postprandial glucose levels after dinner

\section{How might this impact on clinical practice in the foreseeable future?}

- Our findings provide the rationale to further investigate indoor lighting schemes in office and home environments over $24 \mathrm{~h}$ and their potential to prevent or treat insulin resistance

Conclusions/interpretation Together, these findings suggest that the indoor light environment modulates postprandial substrate handling, energy expenditure and thermoregulation of insulin-resistant volunteers in a time-of-day-dependent manner.

Trial registration ClinicalTrials.gov NCT03829982.

Funding We acknowledge the financial support from the Netherlands Cardiovascular Research Initiative: an initiative with support from the Dutch Heart Foundation (CVON2014-02 ENERGISE).

Keywords Biological clock · Circadian rhythm · Glucose intolerance · Insulin resistance · Light at night · Light exposure · Melatonin $\cdot$ Postprandial metabolism $\cdot$ Sleeping metabolic rate

$\begin{array}{ll}\text { Abbreviations } \\ \text { CBT } & \text { Core body temperature } \\ \text { DLMO } & \text { Dim light melatonin onset } \\ \text { DPG } & \text { Distal-proximal skin temperature gradient } \\ \text { E\% } & \text { Energy percentage } \\ \text { LAN } & \text { Light at night } \\ \text { MEQ-SA } & \text { Morningness-Eveningness } \\ & \text { Questionnaire Self-Assessment } \\ \text { REE } & \text { Resting energy expenditure } \\ \text { SMR } & \text { Sleeping metabolic rate } \\ \mathrm{T}_{\text {distal }} & \text { Distal skin temperature } \\ \text { TG } & \text { Triacylglycerol } \\ \mathrm{T}_{\text {proximal }} & \text { Proximal skin temperature }\end{array}$

\section{Abbreviations}

DLMO Dim light melatonin onset

DPG Distal-proximal skin temperature gradient

Energy percentage

LAN Light at night

MEQ-SA Morningness-Eveningness

REE Resting energy expenditure

SMR Sleeping metabolic rate

$\mathrm{T}_{\text {distal }} \quad$ Distal skin temperature

$\mathrm{T}_{\text {proximal }}$ Proximal skin temperature

\section{Introduction}

Light is the most important time cue, i.e. zeitgeber, to synchronise the inner biological clock of mammals to the environmental $24 \mathrm{~h}$ light/dark cycle. The suprachiasmatic nucleus located in the hypothalamus acts as a master pacemaker by integrating the light/dark information received from the retina with endogenously generated rhythms into neural and humoral signals, which in turn can synchronise peripheral clocks in organs such as the liver, skeletal muscle and pancreas [1-3]. In this manner, the organism can anticipate the different 
metabolic demands associated with a particular time slot within the light/dark cycle, such as feeding and fasting, and physical activity and rest. Optimising indoor light conditions to more closely mimic the natural light/dark cycle likely ensures proper regulation of behavioural rhythms, including the sleep/ wake and feeding cycles, and helps in maintaining metabolic health.

However, in our modern society, artificial light is available around the clock and most people expose themselves to electrical light and light-emissive screens during the dark period of the natural light/dark cycle. In humans, the detrimental effects of light at night (LAN) include acutely elevated postprandial glucose and insulin levels [4] and elevated postprandial insulin and glucagon-like peptide-1 levels after breakfast following light exposure during the night [5]. In a prospective cohort study, Obayashi et al [6] found that LAN exposure increases the incidence of type 2 diabetes mellitus in the elderly population. In addition to LAN, our modern society also faces a lack of sufficient time spent under bright light conditions during the daytime $[7,8]$ and, in fact, most time is spent indoors under artificial lighting (under much lower light levels compared with natural daylight outdoors). Bright light exposure before and during breakfast increased postprandial glucose and triacylglycerol (TG) levels in type 2 diabetes mellitus patients, whereas it only increased TG levels in young healthy men [9]. A few studies investigated experimental light exposure protocols over $24 \mathrm{~h}$ and suggested that bright light can influence glucose metabolism in healthy young volunteers at different times of the day when compared with dim light $[10,11]$. However, whether optimising artificial light exposure over a $24 \mathrm{~h}$ period can improve whole-body energy and substrate metabolism and glucose homeostasis in individuals with insulin resistance has not yet been investigated.

Here, we investigated the hypothesis that a combination of bright light during the daytime and dim light during the evening would elicit favourable metabolic effects compared with a combination of dim light during the daytime and bright light during the evening. To this end, we performed $24 \mathrm{~h}$ metabolic phenotyping by means of indirect calorimetry and frequent blood sampling for TG, glucose, insulin and melatonin concentrations upon two different lighting protocols in a randomised crossover design in insulin-resistant volunteers.

\section{Methods}

The study consisted of a randomised crossover protocol with two arms. The Medical Research Ethics Committee of Maastricht University Medical Center approved the study protocol. All participants provided written informed consent before enrolment in the experiment. All procedures were conducted in accordance with the Declaration of Helsinki. Experiments took place between July 2018 and November
2019. The study was registered at ClinicalTrials.gov (registration no. NCT03829982).

Participants Male and female, overweight, insulin-resistant volunteers (40-75 years) were recruited. Insulin resistance was defined as having at least one of the following: (1) impaired fasting glucose: $6.1-6.9 \mathrm{mmol} / \mathrm{l}$; (2) impaired glucose tolerance: $7.8-11.1 \mathrm{mmol} / \mathrm{l} 2 \mathrm{~h}$ after consumption of a $75 \mathrm{~g}$ glucose drink; (3) $\mathrm{HbA}_{1 \mathrm{c}}$ of $39-46 \mathrm{mmol} / \mathrm{mol}$ (5.7$6.4 \%$ ); or (4) low insulin sensitivity defined as a glucose clearance rate $\leq 360 \mathrm{ml} \mathrm{kg}^{-1} \mathrm{~min}^{-1}$ according to the Oral Glucose Insulin Sensitivity model [12]. Participants were non-smokers and generally healthy. Only participants with a habitual bedtime of 23:00 $\mathrm{h} \pm 2 \mathrm{~h}$ and 7-9 $\mathrm{h}$ of sleep per day were included, and participants were excluded if they performed shift work or had travelled across more than one time zone in the 3 months before the study. Additional exclusion criteria were (history of) cardiovascular diseases and regular medication use that could interfere with study outcomes. Using the Morningness-Eveningness Questionnaire Self-Assessment Version 1.3 (MEQ-SA; score: 35-70), extreme early or late chronotypes were excluded. Baseline participant characteristics are shown in Table 1.

Study design All volunteers underwent two $40 \mathrm{~h}$ sessions including two overnight stays in a respiration chamber (detailed study scheme, see Fig. 1). Each session started at 18:00 h on day 1 and ended at 12:00 $\mathrm{h}$ on day 3. In one session (Bright day-Dim evening), participants were exposed to bright light $(1250 \mathrm{~lx})$ during the daytime $(08: 00-18: 00 \mathrm{~h})$ and dim light (5 $\mathrm{lx})$ during the evening (18:00-23:00 h). We

Table 1 Baseline participant characteristics

\begin{tabular}{ll}
\hline Variable & Mean \pm SD \\
\hline Age (years) & $67 \pm 6$ \\
Sex (female/male) & $4 / 10$ \\
Height (m) & $1.72 \pm 0.06$ \\
Body weight (kg) & $88 \pm 12$ \\
BMI (kg/m $\left.{ }^{2}\right)$ & $29.6 \pm 3.0$ \\
Body fat $(\%)$ & $38.4 \pm 6.2$ \\
Habitual sleep duration (h) & $7.9 \pm 0.6$ \\
Habitual bedtime (h) & $23: 34 \pm 00: 45$ \\
MEQ-SA score & $60 \pm 5$ \\
Fasting plasma glucose $(\mathrm{mmol} / \mathrm{l})$ & $5.8 \pm 0.6$ \\
Fasting plasma insulin $(\mathrm{pmol} / \mathrm{l})$ & $80 \pm 52$ \\
2 h plasma glucose $(\mathrm{mmol} / \mathrm{l})$ & $7.5 \pm 2.8$ \\
HbA & \\
$\quad$ mmol $/$ mol & $38 \pm 1$ \\
$\quad \%$ & $5.6 \pm 1.5$ \\
Glucose clearance rate $\left(\mathrm{ml} \mathrm{kg}{ }^{-1} \mathrm{~min}^{-1}\right)$ & $325 \pm 55$ \\
\hline
\end{tabular}




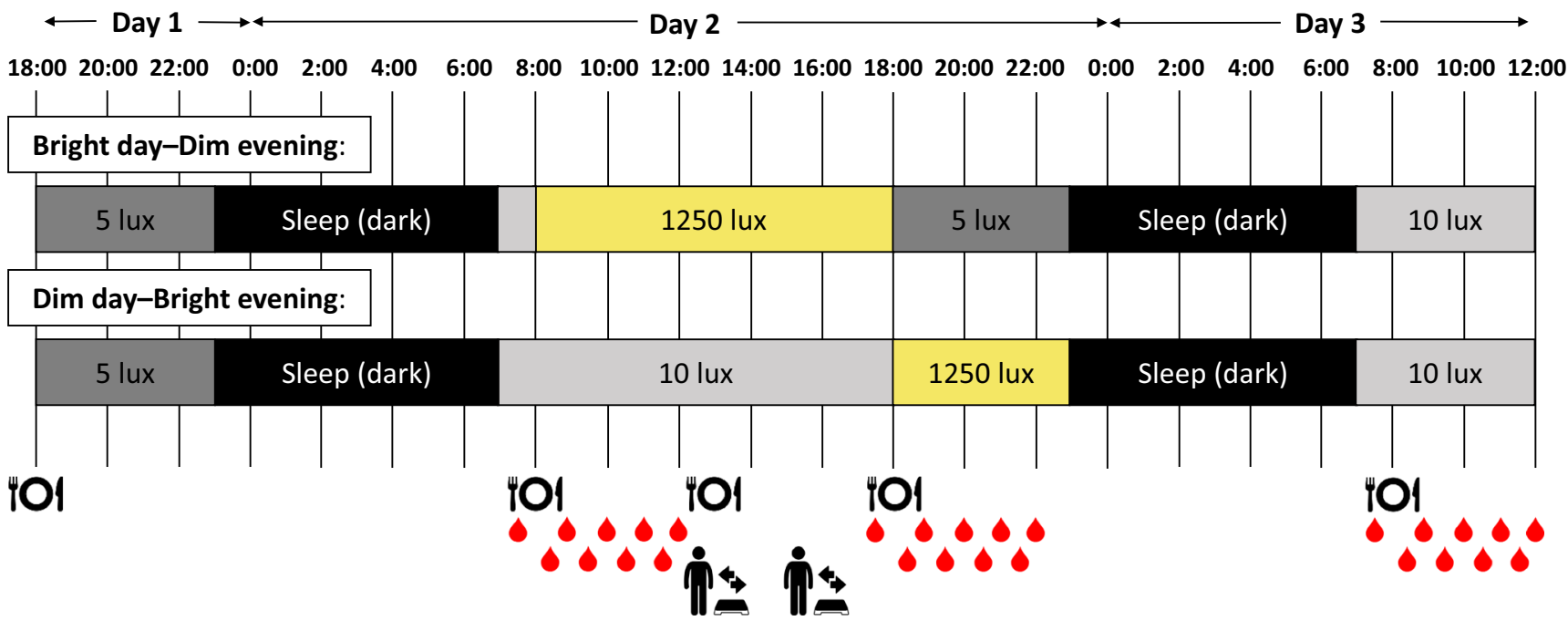

Fig. 1 Study scheme. Fasted blood samples were taken at $07: 45 \mathrm{~h}$ on days 2 and 3 and at 17:45 h on day 2. Postprandial blood samples were taken for $4 \mathrm{~h}$ at $30 \mathrm{~min}$ intervals after both breakfasts and dinner. Slow stepping exercise for $30 \mathrm{~min}$ was performed at 12:30 $\mathrm{h}$ preceding lunch and at 15:30 $\mathrm{h}$

chose $5 \mathrm{~lx}$ for the evening as generally the dim light melatonin onset (DLMO) is determined in dim light below $10 \mathrm{~lx}$. In the other session (Dim day-Bright evening), participants were exposed to dim light (10 lx) during the daytime (08:0018:00 h) and bright light (1250 lx) during the evening (18:00-23:00 h). We chose $10 \mathrm{~lx}$ for the daytime as $5 \mathrm{~lx}$ was considered too low and unpleasant to spend $10 \mathrm{~h}$ in. During the evening of the first day and the morning of the last day of both conditions, the light intensity was low at 5 and $10 \mathrm{~lx}$, respectively. More detailed information on light characteristics is given in electronic supplementary material (ESM) (ESM Methods 'Light characteristics', ESM Table 1, ESM Fig. 1).

For 3 days before each session, participants were instructed to try to sleep every night from $23: 00$ to $07: 00 \mathrm{~h}$, to avoid caffeine and alcohol, to refrain from strenuous exercise and from exposure to excessive bright/dim light, and to follow normal eating patterns (three meals a day at $\sim 08: 00,13: 00$ and 18:00). Participants filled in a food diary over each 3 day run-in period and food intake was kept similar during both sessions. The wash-out period between sessions was at least 4 days and not longer than 4 weeks.

Actigraphy For the entire 3 day pre-period and during their stay in the respiration chamber, participants wore an actigraph (Actiwatch Spectrum, Philips Respironics, Murrysville, PA, USA) on their wrist. Detected bedtime, wakeup time, immobile time during the sleep period (in \%) and activity count were used as variables to: (1) monitor if participants adhered to the instructions (i.e. sleeping times) for the pre-period at home; and to (2) compare activity patterns and sleep characteristics between everyday life at home and under experimental conditions in the respiration chamber.
Energy metabolism and study meals Daily energy expenditure, sleeping metabolic rate (SMR) and substrate oxidation were calculated based on measured concentrations of oxygen consumption and carbon dioxide production (Omnical, Maastricht Instruments, the Netherlands) [13] according to Weir's equations [14]. SMR (defined as lowest $2 \mathrm{~h}$ mean energy expenditure) during the first night was multiplied by an activity factor of 1.5 to calculate energy requirements. Breakfast ( 25 energy percentage [E\%]) was given at 08:00 $\mathrm{h}$, lunch $(30 \mathrm{E} \%)$ at 13:00 $\mathrm{h}$, dinner $(45 \mathrm{E} \%)$ at 18:00 h. Daily macronutrient composition was $\sim 55 \mathrm{E} \%$ as carbohydrates, $\sim 30 \mathrm{E} \%$ as fat ( $\sim 9 \mathrm{E} \%$ saturated) and $\sim 15 \mathrm{E} \%$ as protein. Breakfast and lunch were bread-based, and, thus, the percentage of energy from carbohydrates was higher compared with the dinner. Participants were instructed to finish each meal within 20 min while sitting at a table. No snacks or drinks other than water were provided between meals. The meals were equivalent in macronutrient and caloric content between the two experimental sessions, and it was ensured that participants ate all the provided food in both sessions. In the respiration chamber, the ambient temperature was set to $21^{\circ} \mathrm{C}$ and light exposure was strictly controlled according to the respective protocol. Participants were allowed to perform regular office work and were instructed to follow a daily activity programme (i.e. scheduled standing and stepping) to standardise activity between sessions and participants. The windows in the wall were shielded from the outside to ensure that no light could leak into the room.

Skin and core body temperatures According to ISO standard 9886 [15], skin temperatures were measured at 14 ISOdefined body sites using wireless temperature sensors (iButtons, Maxim Integrated Products, USA) [16]. Core body 
temperature (CBT) was measured using a telemetric pill (Vital Sense, Philips Healthcare, the Netherlands), which was ingested on the first evening after dinner. Participants wore a chest belt that receives and records the core pill's signal (Equivital, Hidalgo, UK).

Body composition Body composition was determined on the last day of the intervention after leaving the respiration chamber at 12:30 h. Body mass and body volume were assessed using air-displacement plethysmography (BodPod, Cosmed, Italy) according to the manufacturer's protocol, as previously reported [17].

Blood sampling and analysis Fasted blood samples were taken via an intravenous cannula placed in the forearm at $07: 45 \mathrm{~h}$ on days 2 and 3 and at 17:45 h on day 2. Postprandial blood samples were taken every $30 \mathrm{~min}$ for $4 \mathrm{~h}$ after both breakfasts and the second dinner (see Fig. 1). Serum TG (Sigma, St Louis, USA) and plasma glucose (Horiba, Montpellier, France) levels were measured colorimetrically using a Cobas Pentra C400 analyser (Horiba, Montpellier, France). Insulin levels were measured in serum with an ELISA kit (Crystal Chem, Elk Grove Village, USA). Melatonin concentration was measured by LC-MS/MS. For detailed information and validation of the method, see van Faassen et al [18]. Intraassay CV was $8.9 \%$ for low concentrations and $3.5 \%$ for high concentrations. Inter-assay CV was $9.5 \%$ for low concentrations and $4.2 \%$ for high concentrations. The lower limit of quantification was $1.9 \mathrm{pg} / \mathrm{ml}(8.18 \mathrm{pmol} / \mathrm{l})$. The DLMO threshold was set to $>10 \mathrm{pg} / \mathrm{ml}(43.05 \mathrm{pmol} / \mathrm{l})$.

Statistical analysis Postprandial data were analysed using a generalised linear mixed model with time and light conditions and their interaction as fixed effects. This model takes missing values into account, which occurred due to the temporary nonfunctioning of the inserted cannula in some individuals $(<5$ individual missing data points for each blood marker out of up to 196 individual data points). Paired $t$ tests were conducted to detect differences between conditions in most outcome measures without repeated measures. The level of significance was set at $\alpha<0.05$ for all analyses. Data are presented as mean \pm SEM if not stated otherwise. All statistical analyses were performed with the GraphPad Prism 8 software package (GraphPad Software, San Diego, USA).

\section{Results}

High compliance of participants to instructions before and during the intervention The mean times participants went to bed and woke up during the 3 day pre-period at home and the 2 intervention days in the respiration chambers were determined based on actigraphy data (ESM Table 2). Activity patterns are shown in ESM Fig. 2. During their stays in the respiration chambers, participants were asked to go to bed at 23:00 $\mathrm{h}$ and wake up at 07:00 $\mathrm{h}$ with lights being switched off and on, respectively. Actigraphy data indicated that participants were lying quietly in bed a few minutes later and were active in the morning already a few minutes earlier. Detected bed and wakeup times did not differ over the 3 day pre-period and the intervention period between conditions. Also, no differences in physical activity were detected between conditions (ESM Fig. 2).

Distal skin temperature is modulated by light exposure Proximal skin temperature ( $\mathrm{T}_{\text {proximal }}$, mean of all measured skin regions of the torso) showed a clear day-night difference throughout the intervention due to the warming effect of the bed sheets covering the skin at night, but was not different between conditions (Fig. 2a). In both conditions, distal skin temperature $\left(\mathrm{T}_{\text {distal }}\right.$, mean of foot and hand temperatures) decreased after waking up, reaching a plateau at $\sim 12: 00 \mathrm{~h}$, which was interrupted by transient increases due to stepping exercise at 12:30 and 15:30 h (Fig. $2 \mathrm{~b}$ ). $\mathrm{T}_{\text {distal }}$ was lower at 18:00 $\mathrm{h}$ when spending the day $(8: 00-18: 00 \mathrm{~h})$ in bright light, compared with spending the day in $\operatorname{dim} \operatorname{light}\left(28.8 \pm 0.3^{\circ} \mathrm{C}\right.$ vs $29.9 \pm 0.4^{\circ} \mathrm{C}, p=0.039$ ). After switching the light conditions at 18:00 h, $\mathrm{T}_{\text {distal }}$ increased and was eventually higher at 23:00 $\mathrm{h}$ in the Bright day-Dim evening condition compared with the Dim day-Bright evening condition $\left(30.1 \pm 0.3^{\circ} \mathrm{C}\right.$ vs $\left.28.8 \pm 0.3^{\circ} \mathrm{C}, p=0.006\right)$. While $\mathrm{T}_{\text {distal }}$ increased over the evening (18:00-23:00 h) in the Bright day-Dim evening condition, it concurrently decreased in the Dim day-Bright evening condition (\% change: $+4.7 \pm 1.3 \%$ vs $-3.4 \pm$ $0.5 \%, p<0.001)$. As $\mathrm{T}_{\text {proximal }}$ was not different between conditions, the distal-proximal skin temperature gradient (DPG) followed the same pattern as $\mathrm{T}_{\text {distal }}$ (Fig. 2c). The DPG, which can be used as an estimate of the degree of vasodilation [19], illustrates a delayed onset of vasodilation in the Dim dayBright evening condition.

Due to technical issues with transmitting the CBT data from the telemetric pill, we obtained complete CBT datasets from only eight volunteers (ESM Fig. 3). The nadir of CBT was not phase shifted either from the first to the second night within any of the two light conditions (Bright day-Dim evening: 0:30 $\pm 17 \mathrm{~min}$ vs 0:32 $\pm 31 \mathrm{~min}, p=0.721$; Dim day-Bright evening: 0:54 $\pm 117 \mathrm{~min}$ and 1:10 $\pm 88 \mathrm{~min}$, $p=0.771$ ) or between light conditions in the second night $(p=0.771)$. No further effect of the light intervention on CBT was detected (condition $\times$ time interaction: $p=0.999$ ).

Plasma melatonin is strongly suppressed by bright evening light In the Dim day-Bright evening condition, plasma melatonin was significantly suppressed compared with Bright dayDim evening (condition $\times$ time interaction: $p<0.001$, condition: $p=0.012$; Fig. 3). Melatonin levels had exceeded the 
Fig. 2 Overview of different skin temperature outcomes averaged over 30 min intervals $(n=13)$. $\mathrm{T}_{\text {proximal }}(\mathbf{a}), \mathrm{T}_{\text {distal }}(\mathbf{b})$ and $\mathrm{DPG}$ (c). The dashed lines with the light bulb indicate the time points when the light settings were changed. Data are presented as mean \pm SEM
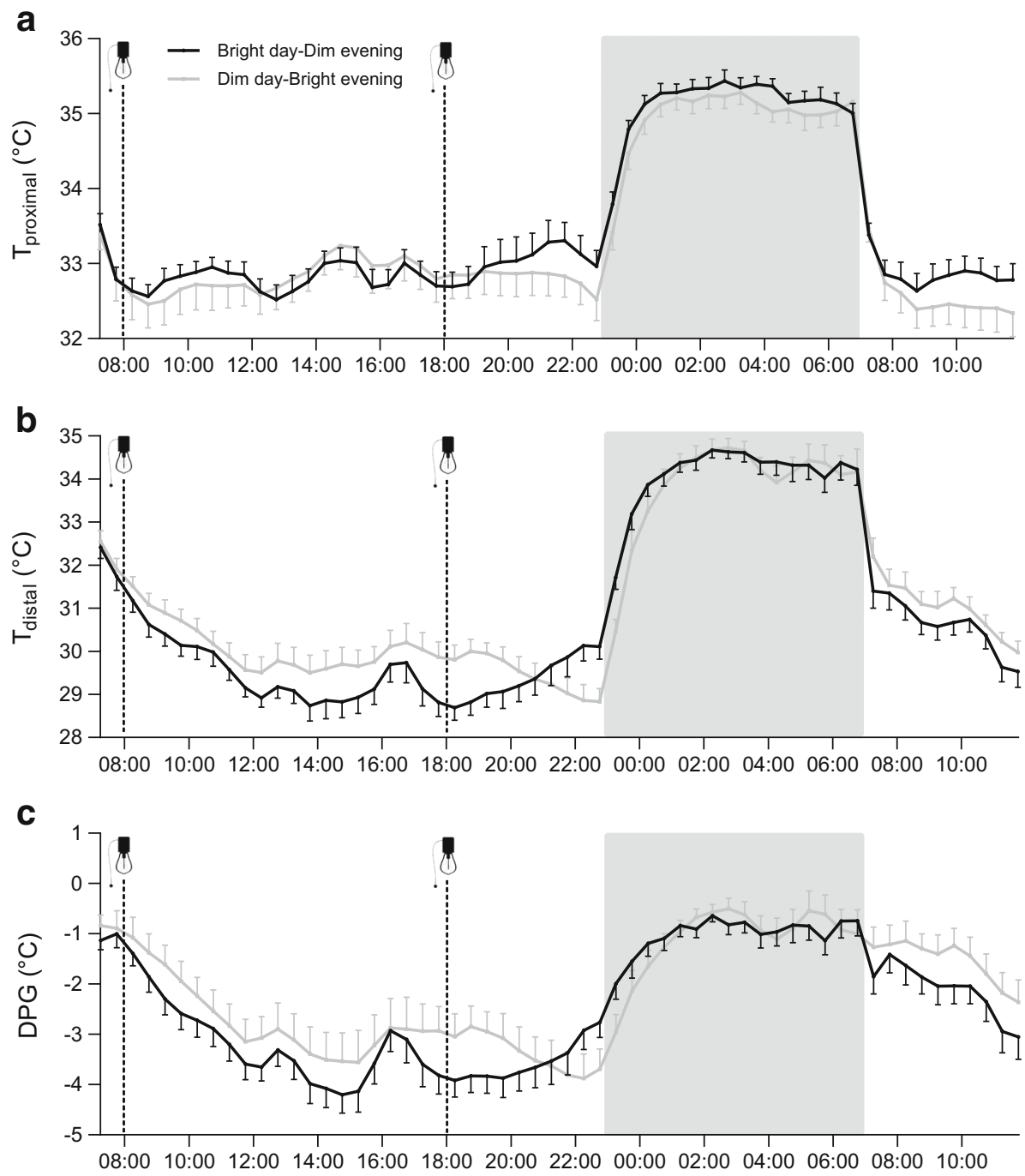

DLMO criterion of $>10 \mathrm{pg} / \mathrm{ml}(43.05 \mathrm{pmol} / \mathrm{l})$ in seven participants at 21:30 $\mathrm{h}$ upon Bright day-Dim evening (20.1 \pm $6.3 \mathrm{pg} / \mathrm{ml}[86.14 \pm 27.12 \mathrm{pmol} / \mathrm{l}], n=11$; Fig. 3a). Only two participants exceeded DLMO levels already at 20:30 h

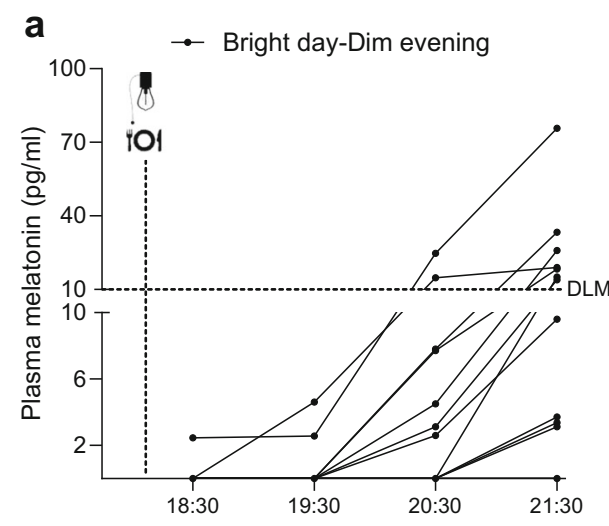

Fig. 3 Plasma melatonin $(n=14)$ in the evening of day 2 upon Bright day-Dim evening (a) and Dim day-Bright evening (b). Lines represent individual data. Data points below the detection threshold of $1.9 \mathrm{pg} / \mathrm{ml}$ and the remaining five at 21:30 $\mathrm{h}$. In contrast, upon Dim dayBright evening, melatonin concentrations that exceeded the detection threshold of $1.9 \mathrm{pg} / \mathrm{ml}(8.18 \mathrm{pmol} / \mathrm{l})$ could be detected in only four participants (Fig. 3b).

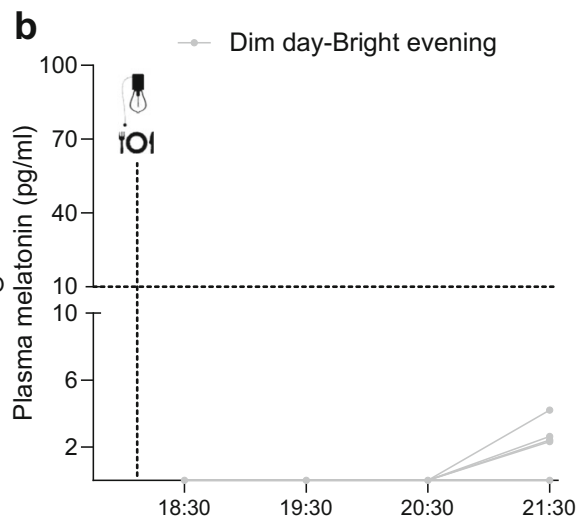

$(8.18 \mathrm{pmol} / \mathrm{l})$ are illustrated as 0 values. The horizontal dashed line indicates the DLMO threshold of $10 \mathrm{pg} / \mathrm{ml}$ (43.05 pmol/l). To convert melatonin values from $\mathrm{pg} / \mathrm{ml}$ to $\mathrm{pmol} / \mathrm{l}$, please multiply by 4.305 
Evening light exposure modulates postprandial glucose In the Bright day-Dim evening condition, postprandial plasma glucose after the first breakfast increased more compared with the Dim day-Bright evening condition, although this did not reach statistical significance (condition $\times$ time interaction: $p=0.097$; Fig. 4a). In addition, plasma TG after the first breakfast increased significantly more in the Bright day-Dim evening condition (condition $\times$ time interaction: $p=0.029$; Fig. $4 \mathrm{~b}$ ) as compared with the Dim day-Bright evening condition.

In the Bright day-Dim evening condition, plasma glucose was lower at 17:45 h, just before serving dinner and activating the evening light settings, compared with in the Dim dayBright evening condition $(5.0 \pm 0.2$ vs $5.2 \pm 0.2 \mathrm{mmol} / \mathrm{l}$, $p=0.02$ ). In the Bright day-Dim evening condition, glucose levels after dinner increased more compared with the Dim day-Bright evening condition (condition $\times$ time interaction: $p<0.001$; Fig. 4c), with a higher incremental AUC (394 \pm 66 vs $307 \pm 55, p=0.009$ ). Plasma insulin and TG levels after dinner did not differ between conditions (condition $\times$ time interaction: $p=0.993$ and $p=0.663$; Fig. $4 \mathrm{~d}, \mathrm{e})$.

On the last day of the intervention, after an overnight fast and under standardised dim light conditions, all plasma metabolites before and after breakfast were not differently affected by the prior differences in light exposure (Fig. $4 \mathrm{f}-\mathrm{h}$ ).

The Dim day-Bright evening condition reduced the SMR and energy expenditure after dinner The $24 \mathrm{~h}$ energy expenditure on day 2 (07:00 h on day 2 to 07:00 h on day 3, Bright day-Dim evening: 10,124 $\pm 394 \mathrm{~kJ}$, Dim day-Bright evening: 10,036 \pm $364 \mathrm{~kJ} ; p=0.446)$ and the energy expenditure on day 3 (07:00 12:00 h, Bright day-Dim evening: $1884 \pm 80 \mathrm{~kJ}$, Dim dayBright evening: $1909 \pm 80 \mathrm{~kJ} ; p=0.558$ ) were not significantly different between light conditions (Fig. 5a). Furthermore, no differences between conditions could be detected in energy
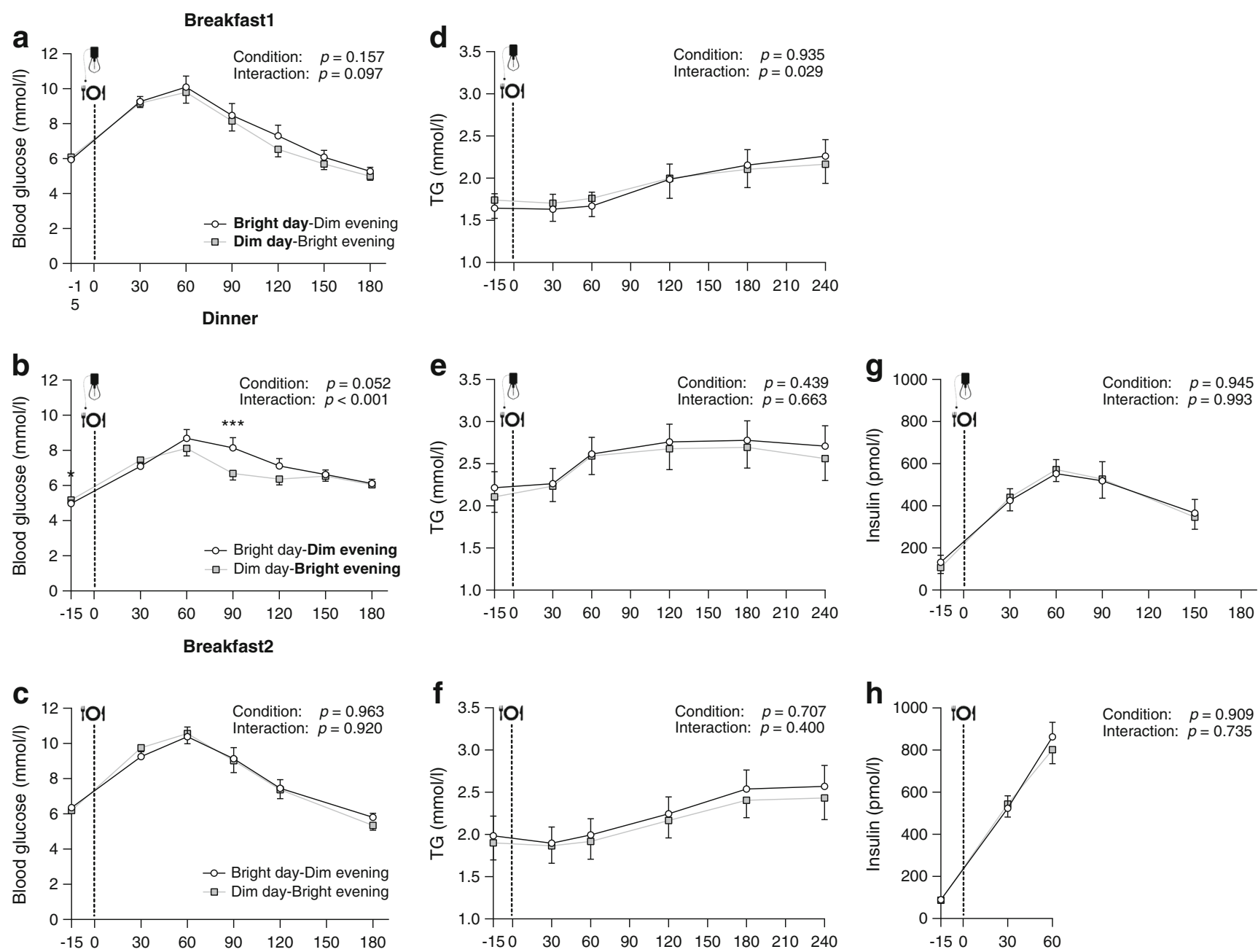

Fig. 4 Postprandial plasma responses for the two meals on day 2 (Breakfast1 $[n=13]$ and Dinner $[n=14]$ ) and breakfast on day 3 (Breakfast2 $[n=13]$ ). Blood glucose (a) and TG (d) for the first breakfast; blood glucose (b), TG (e) and insulin (g) for the dinner; blood glucose (c), TG (f) and insulin (h) for the second breakfast.

Postprandial data were analysed using a generalised linear mixed model with time and light conditions and their interaction as fixed effects. Data are presented as mean $\pm \mathrm{SEM}$; $* * * p<0.001,{ }^{*} p<0.05$ (note that there is a single * symbol in Fig. 4b, partially obscured by the $y$-axis) 

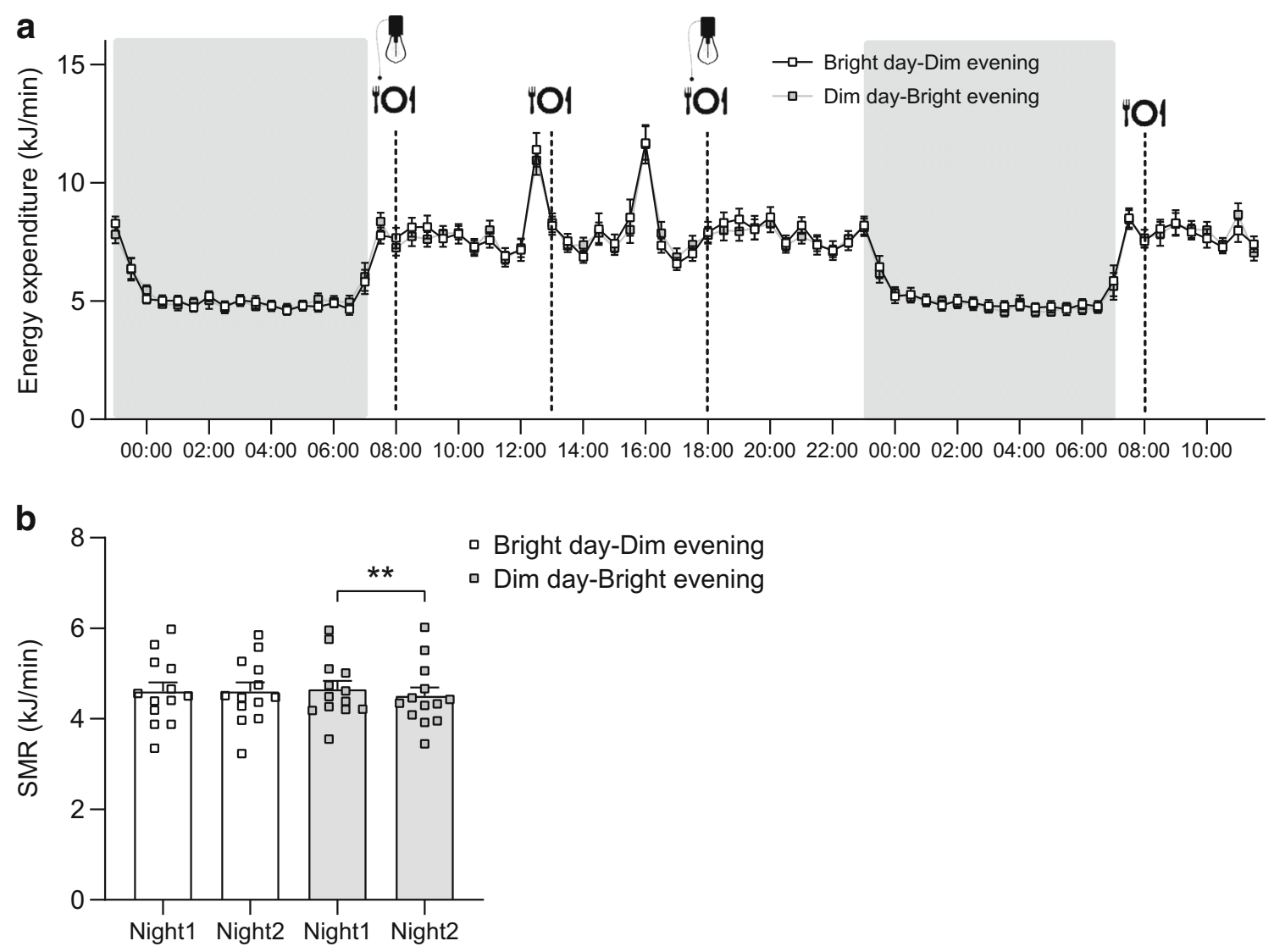

Fig. 5 Energy expenditure over the entire time spent in the respiration chamber (a) and SMR of both nights per condition ( $n=13$; Night1 refers to the first baseline night spent in the respiration chamber without any

expenditure in the postprandial phase of the first breakfast (08:00-11:00 h: $1407 \pm 63 \mathrm{~kJ}$ vs $1398 \pm 54 \mathrm{~kJ}, p=0.751$ ). However, in the postprandial phase after dinner, energy expenditure was higher in the Bright day-Dim evening condition (18:00-21:00 h: $1482 \pm 63 \mathrm{~kJ}$ vs $1432 \pm 63 \mathrm{~kJ}, p=0.044)$. The SMR was reduced in the night after the light intervention compared with the night preceding the light intervention, but only after Dim day-Bright evening $(-3.3 \pm 0.9 \%$, condition $\times$ time interaction: $p=0.013, n=13$; Fig. $5 b$ ). The SMR of the night after the light intervention was higher in the Bright day-Dim evening condition compared with the Dim dayBright evening condition, although the difference was not significant ( $4.6 \pm 0.2$ vs $4.5 \pm 0.2 \mathrm{~kJ} / \mathrm{min}, p=0.065)$. The respiratory exchange ratio, calculated as the quotient of $\mathrm{CO}_{2}$ production and $\mathrm{O}_{2}$ consumption, was not different between conditions over any specific time interval $(\geq 1 \mathrm{~h}$ ) of the $40 \mathrm{~h}$ spent in the respiration chamber (ESM Fig. 4).

\section{Discussion}

Optimising indoor light conditions to more closely mimic the natural light/dark cycle holds promise to prevent differences in light intervention; Night2 refers to the second night after the respective light intervention) (b); $p$ values are based on paired $t$ tests; $* * p<0.01$. Data are presented as mean \pm SEM

adverse effects on whole-body energy and glucose metabolism associated with the nowadays prevailing constant indoor lighting conditions. Here, we found that spending the day in bright light led to lower plasma glucose levels preceding the last meal of the day, whereas plasma glucose levels after that meal were higher after the Bright day-Dim evening condition, i.e. when the dinner was consumed under dim light conditions. The Bright day-Dim evening condition had a positive influence on energy expenditure: the SMR could be maintained compared with the night preceding the light intervention, whereas it dropped after the Dim day-Bright evening condition. Moreover, energy expenditure during dinner was increased in the Bright dayDim evening condition. As expected, Bright day-Dim evening facilitated melatonin secretion in the evening, which was strongly suppressed in the Dim day-Bright evening condition. Furthermore, we found a lower $\mathrm{T}_{\text {distal }}$ at 18:00 $\mathrm{h}$ after spending the day in bright light and higher $\mathrm{T}_{\text {distal }}$ after spending the evening in dim light at 23:00 $\mathrm{h}$ (Bright day-Dim evening). Postprandial plasma TG levels were higher when breakfast was consumed under bright light conditions. Together, these findings suggest that the indoor light environment of insulin-resistant individuals 
can modulate metabolic variables in a time-of-daydependent manner to influence metabolic health in the long term.

The role of melatonin as a mediator between environmental light and mammalian metabolism is well established. Melatonin secretion by the pineal gland requires darkness after sunset, with the inhibitory signal for lightinduced melatonin suppression ultimately coming from intrinsically photosensitive retinal ganglion cells [20, 21]. The suppression of melatonin upon bright light during the evening in the present study is hence not surprising. Melatonin started to rise $2-4 \mathrm{~h}$ before habitual bedtime upon Bright day-Dim evening, which is in line with the reported range of DLMO in young healthy volunteers [22-24]. In contrast, CBT data $(n=8)$ consistently indicated a relatively early occurrence of the CBT nadir (range of the mean of all nights between 00:00 and 01:15 h) in line with another study in insulin-resistant men of similar age [25], which seems to be phaseadvanced compared with younger participants (i.e. between 04:00 and 06:00 h [26, 27]). Also, chronotype assessment characterised our volunteers as being of moderate morning type on average (MEQ-SA: $60 \pm 5$ ) which is typical in the aged population [28].

The interplay between melatonin and human glucose metabolism has recently gained a lot of interest [29] due to evidence for adverse effects of circadian misalignment (e.g. night-shift work) on glucose metabolism $[26,30]$ and the discovery of type 2 diabetes mellitus risk variants in the melatonin receptor $1 \mathrm{~B}$ gene, i.e. MTNR1B $[31,32]$. MTNR1B has even been found to be a predictor for the transition from normoglycaemia to glucose intolerance [33]. Moreover, melatonin supplementation in the morning has been shown to acutely reduce glucose tolerance in aged women [34]. In the present study, we observed higher postprandial glucose levels after dinner in the Bright day-Dim evening condition compared with Dim day-Bright evening, with the greatest difference $90 \mathrm{~min}$ after the meal, at 19:30 h. As melatonin was strongly suppressed by bright light in the evening, one could assume that the suppression of melatonin was beneficial for glucose homeostasis. However, it should be noted that under dim light conditions, melatonin onset occurred for most volunteers at 20:30-21:30 h, whereas the light effects on postprandial glucose occurred earlier. Therefore, the effects of the light condition on postprandial glucose can probably not be explained by its effect on melatonin.

The reduced postprandial glucose levels after the dinner meal in the Dim day-Bright evening condition occurred also in the absence of differences in insulin levels. Sone et al [35] demonstrated that gastric motility and carbohydrate absorption (determined by breath hydrogen excretion) were both decreased in young women consuming an evening meal at 17:00 $\mathrm{h}$ after spending the day from $07: 00$ to $15: 00 \mathrm{~h}$ in dim light $(80 \mathrm{~lx})$ compared with bright light $(5000 \mathrm{~lx})$. These effects were accompanied by higher parasympathetic activity in the evening after spending the day in bright light, which is likely to promote gut functioning and thereby absorption of carbohydrates [36]. In support, Tokura et al [37] found that secretion of saliva in response to food stimulation was influenced by the light environment in a time-of-day-dependent manner, with enhanced saliva secretion after bright light during the daytime and suppressed secretion after bright light in the evening. Enhanced secretion of saliva facilitates subsequent gastrointestinal digestion of complex carbohydrates through the presence of salivary amylase. Taken together, combining bright light during the daytime and dim light during the evening may promote digestion of food and could contribute to our findings of increased postprandial glucose levels after the dinner meal. Future research integrating appropriate methodology such as a meal tracer to quantify gastrointestinal processes is required to further support this claim. Albreiki et al [4] only investigated the acute metabolic effects of LAN in young healthy volunteers without taking prior light exposure during the daytime into account, and found, in contrast to our findings, negative effects of LAN on glucose tolerance. However, in that study, the meal challenge was also given later at 22:00 h. In line with Albreiki et al [4], Cheung et al [11] showed that both morning and evening blueenriched light exposure (for $3 \mathrm{~h}$ during mealtime) increased postprandial insulin levels and HOMA-IR compared with a constant $24 \mathrm{~h} \mathrm{dim}$ light condition in young healthy volunteers. In the present study, the abrupt switch of the light conditions at 18:00 $\mathrm{h}$ from bright to dim or vice versa, which precisely coincided with dinner time, could explain why our results contradict previous reports. Ultimately, how LAN influences glucose homeostasis may depend on prior light exposure before giving the meal challenge, how late into the evening food intake takes place, and the state of glucose tolerance and age of the studied population.

A time-of-day dependency of the effects of light exposure on glucose metabolism becomes evident when considering that we found the opposite pattern in the morning, with a tendency towards a greater increase in postprandial glucose upon bright light in the morning during/after breakfast, although this did not reach statistical significance. In comparison with Versteeg et al [9], who found significantly increased postprandial glucose levels in type 2 diabetes mellitus patients who were exposed to a brighter morning light stimulus (i.e. $4000 \mathrm{~lx}$ vs $1250 \mathrm{~lx}$ in our study), our data now suggest that either higher brightness levels are needed to modulate the postprandial glucose response after breakfast, or the modulatory effect depends on the severity of glucose intolerance of the studied population. In line with Versteeg et al [9], our data confirm that postprandial TG levels after breakfast are consistently increased upon bright light in the morning. 
In accordance with earlier findings in young healthy individuals $[38,39]$, we found that the environmental light strongly modulates $T_{\text {distal }}$ also in insulin-resistant volunteers. $T_{\text {distal }}$ is lowered by vasoconstriction of the peripheral skin and can serve to increase CBT. Our data suggest that this effect was potentiated upon bright light during the daytime, whereas vasodilation to lower CBT, which promotes a rapid onset of sleep [19], was only facilitated upon dim light during the evening. Vasodilation upon dim light in the evening is mostly mediated through reduction of the sympathetic vasoconstrictor tonus [40]. Overall, Bright day-Dim evening induced a diurnal pattern in $\mathrm{T}_{\text {distal }}$ and the DPG. This supports a healthy diurnal variation in blood pressure with higher values during the daytime and lower values at night [41-43]. Therefore, the combination of bright light during the daytime and dim light during the evening seems promising to improve diurnal variation in blood pressure in a population that is prone to developing cardiovascular complications in the long term.

A novel finding of our study is that prior light exposure can influence the SMR. When comparing the night following the Dim day-Bright evening scheme compared with the previous night, SMR dropped by $3.3 \pm 0.9 \%$. Following the Bright day-Dim evening scheme, the SMR was maintained. Previous studies have found that resting energy expenditure (REE; i.e. assessed during wakefulness in contrast to SMR) shows circadian rhythmicity [44], and that circadian misalignment, such as sleep restriction and night-shift work, can alter energy expenditure $[26,45,46]$. Since light exposure acts as a zeitgeber to align endogenous physiological rhythms to the environmental light/dark cycle, it is tempting to speculate that light exposure may misalign the diurnal variation in REE. More research is needed to investigate how light exposure could affect energy expenditure, especially since small changes in energy metabolism can contribute to the development of obesity and metabolic diseases.

In conclusion, by performing detailed $24 \mathrm{~h}$ metabolic phenotyping by means of frequent blood sampling, continuous indirect calorimetry and skin temperature assessment, we demonstrate that the timing of light exposure can influence postprandial substrate handling, energy expenditure and thermoregulation of insulin-resistant individuals. In contrast to the Dim day-Bright evening condition, the Bright day-Dim evening condition mostly elicited favourable outcomes: lower plasma glucose levels preceding the dinner meal, greater energy expenditure in response to the dinner meal, conservation of SMR and facilitation of a diurnal pattern in $\mathrm{T}_{\text {distal. }}$. The finding that postprandial $\mathrm{TG}$ levels after breakfast and postprandial glucose levels after dinner were less increased in the Dim day-Bright evening condition warrants further investigation. In the future, more research is required to exploit different light regimens in office buildings and home settings for their potential to prevent metabolic diseases.
Supplementary Information The online version contains peer-reviewed but unedited supplementary material available at https://doi.org/10.1007/ s00125-021-05643-9.

Acknowledgements P. Schoffelen and M. Souren are gratefully acknowledged for their technical assistance.

Data availability The datasets generated during and/or analysed during the current study are available from the corresponding author on reasonable request.

Funding We acknowledge the financial support from the Netherlands Cardiovascular Research Initiative: an initiative with support from the Dutch Heart Foundation (CVON2014-02 ENERGISE).

Authors' relationships and activities The authors declare that there are no relationships or activities that might bias, or be perceived to bias, their work.

Contribution statement J-FH, JW, DD, PR, BD, DvM and EM-K were involved in data acquisition and analysis. J-FH and JW wrote the manuscript, while all other authors critically revised the manuscript. LS, JH, MCMG, WvML and PS contributed to the concept design and interpretation of data. All authors approved the final version to be published. PS is the guarantor of this work.

Open Access This article is licensed under a Creative Commons Attribution 4.0 International License, which permits use, sharing, adaptation, distribution and reproduction in any medium or format, as long as you give appropriate credit to the original author(s) and the source, provide a link to the Creative Commons licence, and indicate if changes were made. The images or other third party material in this article are included in the article's Creative Commons licence, unless indicated otherwise in a credit line to the material. If material is not included in the article's Creative Commons licence and your intended use is not permitted by statutory regulation or exceeds the permitted use, you will need to obtain permission directly from the copyright holder. To view a copy of this licence, visit http://creativecommons.org/licenses/by/4.0/.

\section{References}

1. Reinke H, Asher G (2019) Crosstalk between metabolism and circadian clocks. Nat Rev Mol Cell Biol 20(4):227-241. https:// doi.org/10.1038/s41580-018-0096-9

2. Gutierrez-Monreal MA, Harmsen J-F, Schrauwen P, Esser KA (2020) Ticking for metabolic health: the skeletal-muscle clocks. Obesity 28(Suppl 1):S46-S54

3. Mason IC, Qian J, Adler GK, Scheer FAJL (2020) Impact of circadian disruption on glucose metabolism: implications for type 2 diabetes. Diabetologia 63(3):462-472. https://doi.org/10.1007/ s00125-019-05059-6

4. Albreiki MS, Middleton B, Hampton SM (2017) A single night light exposure acutely alters hormonal and metabolic responses in healthy participants. Endocr Connect 6(2):100-110. https://doi.org/ 10.1530/EC-16-0097

5. Gil-Lozano M, Hunter PM, Behan L-A, Gladanac B, Casper RF, Brubaker PL (2016) Short-term sleep deprivation with nocturnal light exposure alters time-dependent glucagon-like peptide-1 and insulin secretion in male volunteers. Am J Physiol Endocrinol Metab 310(1):E41-E50. https://doi.org/10.1152/ajpendo.00298. 2015 
6. Obayashi K, Yamagami Y, Kurumatani N, Saeki K (2020) Bedroom lighting environment and incident diabetes mellitus: a longitudinal study of the HEIJO-KYO cohort. Sleep Med 65:1-3. https://doi.org/10.1016/j.sleep.2019.07.006

7. Münch M, Wirz-Justice A, Brown SA et al (2020) The role of daylight for humans: gaps in current knowledge. Clocks \& Sleep 2(1):61-85. https://doi.org/10.3390/clockssleep2010008

8. Daugaard S, Markvart J, Bonde JP et al (2019) Light exposure during days with night, outdoor, and indoor work. Ann Work Expo Health 63(6):651-665. https://doi.org/10.1093/annweh/ wxy110

9. Versteeg RI, Stenvers DJ, Visintainer D et al (2017) Acute effects of morning light on plasma glucose and triglycerides in healthy men and men with type 2 diabetes. J Biol Rhythm 32(2):130-142. https://doi.org/10.1177/0748730417693480

10. Melanson EL, Ritchie HK, Dear TB et al (2018) Daytime bright light exposure, metabolism, and individual differences in wake and sleep energy expenditure during circadian entrainment and misalignment. Neurobiol Sleep Circadian Rhythms 4:49-56. https://doi.org/10.1016/j.nbscr.2017.12.002

11. Cheung IN, Zee PC, Shalman D, Malkani RG, Kang J, Reid KJ (2016) Morning and evening blue-enriched light exposure alters metabolic function in Normal weight adults. PLoS One 11(5): e0155601. https://doi.org/10.1371/journal.pone.0155601

12. Mari A, Pacini G, Murphy E, Ludvik B, Nolan JJ (2001) A modelbased method for assessing insulin sensitivity from the oral glucose tolerance test. Diabetes Care 24(3):539-548. https://doi.org/10. 2337/diacare.24.3.539

13. Schoffelen PF, Westerterp KR, Saris WH, Ten Hoor F (1997) A dual-respiration chamber system with automated calibration. J Appl Physiol 83(6):2064-2072. https://doi.org/10.1152/jappl.1997.83.6. 2064

14. Weir JBDEB (1949) New methods for calculating metabolic rate with special reference to protein metabolism. J Physiol 109(1-2):1-9

15. ISO (2004) ISO 9886: 2004. Ergonomics-evaluation of thermal strain by physiological measurements. International Standards Organization, Geneva

16. van Marken Lichtenbelt WD, Daanen HAM, Wouters L et al (2006) Evaluation of wireless determination of skin temperature using iButtons. Physiol Behav 88(4-5):489-497

17. Plasqui G, Soenen S, Westerterp-Plantenga MS, Westerterp KR (2011) Measurement of longitudinal changes in body composition during weight loss and maintenance in overweight and obese subjects using air-displacement plethysmography in comparison with the deuterium dilution technique. Int J Obes 35(8):11241130. https://doi.org/10.1038/ijo.2010.250

18. van Faassen M, Bischoff R, Kema IP (2017) Relationship between plasma and salivary melatonin and cortisol investigated by LC-MS/ MS. Clin Chem Lab Med 55(9):1340-1348. https://doi.org/10. 1515/cclm-2016-0817

19. Kräuchi K, Cajochen C, Werth E, Wirz-Justice A (2000) Functional link between distal vasodilation and sleep-onset latency? Am J Physiol Regul Integr Comp Physiol 278(3):R741-R748. https:// doi.org/10.1152/ajpregu.2000.278.3.R741

20. Berson DM, Dunn FA, Takao M (2002) Phototransduction by retinal ganglion cells that set the circadian clock. Science 295(5557): 1070-1073. https://doi.org/10.1126/science.1067262

21. Barnard AR, Hattar S, Hankins MW, Lucas RJ (2006) Melanopsin regulates visual processing in the mouse retina. Curr Biol 16(4): 389-395. https://doi.org/10.1016/j.cub.2005.12.045

22. Santhi N, Thorne HC, van der Veen DR et al (2012) The spectral composition of evening light and individual differences in the suppression of melatonin and delay of sleep in humans. J Pineal Res 53(1):47-59. https://doi.org/10.1111/j.1600-079X.2011. 00970.x
23. Wright KP Jr, Gronfier C, Duffy JF, Czeisler CA (2005) Intrinsic period and light intensity determine the phase relationship between melatonin and sleep in humans. J Biol Rhythm 20(2):168-177. https://doi.org/10.1177/0748730404274265

24. Zerbini G, Winnebeck EC, Merrow M (2021) Weekly, seasonal, and chronotype-dependent variation of dim-light melatonin onset. J Pineal Res 70(3):e12723. https://doi.org/10.1111/jpi.12723

25. Wefers J, Connell NJ, Fealy CE et al (2020) Day-night rhythm of skeletal muscle metabolism is disturbed in older, metabolically compromised individuals. Mol Metab 41:101050. https://doi.org/ 10.1016/j.molmet.2020.101050

26. Wefers J, van Moorsel D, Hansen J et al (2018) Circadian misalignment induces fatty acid metabolism gene profiles and compromises insulin sensitivity in human skeletal muscle. Proc Natl Acad Sci U S A 115(30):7789-779427. https://doi.org/10.1073/pnas. 1722295115

27. Refinetti R, Menaker M (1992) The circadian rhythm of body temperature. Physiol Behav 51(3):613-637. https://doi.org/10. 1016/0031-9384(92)90188-8

28. Hood S, Amir S (2017) The aging clock: circadian rhythms and later life. J Clin Invest 127(2):437-446. https://doi.org/10.1172/ JCI90328

29. Garaulet M, Qian J, Florez JC, Arendt J, Saxena R, Scheer FAJL (2020) Melatonin effects on glucose metabolism: time to unlock the controversy. Trends Endocrinol Metab 31(3):192-204. https://doi. org/10.1016/j.tem.2019.11.011

30. Morris CJ, Yang JN, Garcia JI et al (2015) Endogenous circadian system and circadian misalignment impact glucose tolerance via separate mechanisms in humans. Proc Natl Acad Sci U S A 112(17):E2225-E2234. https://doi.org/10.1073/pnas.1418955112

31. Bonnefond A, Clément N, Fawcett K et al (2012) Rare MTNR1B variants impairing melatonin receptor $1 \mathrm{~B}$ function contribute to type 2 diabetes. Nat Genet 44(3):297-301. https://doi.org/10. 1038/ng.1053

32. Tuomi T, Nagorny CLF, Singh P et al (2016) Increased melatonin signaling is a risk factor for type 2 diabetes. Cell Metab 23(6):10671077. https://doi.org/10.1016/j.cmet.2016.04.009

33. Walford GA, Green T, Neale B et al (2012) Common genetic variants differentially influence the transition from clinically defined states of fasting glucose metabolism. Diabetologia 55(2):331-339. https://doi.org/10.1007/s00125-011-2353-8

34. Cagnacci A, Arangino S, Renzi A et al (2001) Influence of melatonin administration on glucose tolerance and insulin sensitivity of postmenopausal women. Clin Endocrinol 54(3):339-346. https:// doi.org/10.1046/j.1365-2265.2001.01232.x

35. Sone Y, Hyun K-J, Nishimura S, Lee Y-A, Tokura H (2003) Effects of $\mathrm{dim}$ or bright-light exposure during the daytime on human gastrointestinal activity. Chronobiol Int 20(1):123-133

36. Nishimura S, Hyun K-J, Lee Y-A, Tokura H (2003) Increase in parasympathetic nerve activity during the nighttime following bright light exposure during the daytime. Biol Rhythm Res 34(3):233-240

37. Tokura H, Gotoh K, Kondo M, Akimoto T, Kanikowska D (2002) Salivary secretion under the influence of bright/dim light exposure in the morning and evening in humans. Biol Rhythm Res 33(2):129-140

38. Cajochen C, Münch M, Kobialka S et al (2005) High sensitivity of human melatonin, alertness, thermoregulation, and heart rate to short wavelength light. J Clin Endocrinol Metab 90(3):13111316. https://doi.org/10.1210/jc.2004-0957

39. Lok R, van Koningsveld MJ, Gordijn MCM, Beersma DGM, Hut RA (2019) Daytime melatonin and light independently affect human alertness and body temperature. J Pineal Res 67(1): e12583. https://doi.org/10.1111/jpi.12583 
40. Kräuchi K (2002) How is the circadian rhythm of core body temperature regulated? Clin Auton Res 12(3):147-149. https:// doi.org/10.1007/s10286-002-0043-9

41. Kräuchi K, Gompper B, Hauenstein D et al (2012) Diurnal blood pressure variations are associated with changes in distal-proximal skin temperature gradient. Chronobiol Int 29(9):1273-1283. https:// doi.org/10.3109/07420528.2012.719961

42. Shea SA, Hilton MF, Hu K, Scheer FAJL (2011) Existence of an endogenous circadian blood pressure rhythm in humans that peaks in the evening. Circ Res 108(8):980-984. https://doi.org/10.1161/ CIRCRESAHA.110.233668

43. Salles GF, Reboldi G, Fagard RH et al (2016) Prognostic effect of the nocturnal blood pressure fall in hypertensive patients. Hypertension 67(4):693-700. https://doi.org/10.1161/ HYPERTENSIONAHA.115.06981
44. Zitting K-M, Vujovic N, Yuan RK et al (2018) Human resting energy expenditure varies with circadian phase. Curr Biol 28(22): 3685-3690.e3. https://doi.org/10.1016/j.cub.2018.10.005

45. Buxton OM, Cain SW, O'Connor SP et al (2012) Adverse metabolic consequences in humans of prolonged sleep restriction combined with circadian disruption. Sci Transl Med 4(129): 129ra43. https://doi.org/10.1126/scitranslmed.3003200

46. McHill AW, Melanson EL, Higgins J et al (2014) Impact of circadian misalignment on energy metabolism during simulated nightshift work. Proc Natl Acad Sci U S A 111(48):1730217307. https://doi.org/10.1073/pnas.1412021111

Publisher's note Springer Nature remains neutral with regard to jurisdictional claims in published maps and institutional affiliations. 